\title{
Utilidad del análisis de flujo por videoangiografía ICG (flow 800) en neurocirugía vascular. Nota técnica
}

\author{
Usefulness of flow 800 icg videoangiography in vascular \\ neurosurgery. Technical note
}

\author{
José Luis Cuevas Seguel', Pablo Carmona Rammsy', Benjamín Abarca Carrasco'
}

${ }^{1}$ Neurocirujano. Equipo de Neurocirugía Vascular y Base del Cráneo. Servicio de Neurocirugía, Hospital de Puerto Montt, Chile.

\section{Resumen}

Introducción: La videoangiografía con indocianina verde (ICG) es una herramienta que ha tenido gran impacto neurocirugía vascular. La integración de un nuevo software (FLOW 800 Carls Zeiss) permite un análisis semicuantitativo del flujo sanguíneo que mejora nuestro entendimiento de la fisiopatología arterial, venosa y parenquimatosa durante el acto quirúrgico. Objetivo: Describir la utilidad del análisis de flujo por videoangiografía ICG (FLOW 800) en cirugía vascular cerebral. Método: Se presentan dos casos en los cuales el análisis de flujo fue determinante para la toma de decisiones durante la cirugía. EI primer caso corresponde a un paciente intervenido quirúrgicamente por malformación arteriovenosa (MAV). El segundo caso corresponde a un paciente con isquemia cerebral crónica en quien se realizó bypass cerebral. Resultados: El análisis de flujo por videoangiografía-ICG (FLOW 800) demostró ser útil para la identificación de vasos aferentes y eferentes en MAV de nido complejo. En cirugía de bypass permitió una mejor selección de arteria receptora, así como verificar la mejoría en la perfusión parenquimatosa post revascularización. Conclusiones: El análisis de flujo (FLOW 800) es una herramienta eficiente y no invasiva, que provee de información valiosa para la toma de decisiones en neurocirugía vascular.

Palabras clave: Cerebral revascularization, cerebral bypass, videoangiography, ICG, indocyanine green, software analysis; FLOW 800.

\section{Abstract}

Background: Indocyanine green (ICG) videography has had a great impact on vascular neurosurgery. The integration of a new software (FLOW 800 Carls Zeiss) has allowed the performance of a semiquantitative flow analysis that has improved our understanding of the pathophysiology of arterial, venous, and parenchymal blood flow in neurosurgical procedures. Objective: To describe the utility of FLOW 800 ICG videoangiography in cerebrovascular surgery. Method: Two cases are presented in which the flow analysis was decisive for the decision making during the surgery. The first case corresponds to a patient surgically treated for arteriovenous malformation (AVM). The second case corresponds to a patient with chronic cerebral ischemia in whom cerebral bypass was performed. Results: FLOW 800 ICG videoangiography analysis proved to be useful for the identification of afferent and efferent vessels in a complex nidus AVM. In bypass surgery allowed a better selection of recipient artery, as well as to verify the improvement in parenchymal perfusion after revascularization. Conclusion: FLOW 800 is an efficient and non-invasive tool that provides valuable information for decision making in vascular neurosurgery.

Key words: Cerebral revascularization, cerebral bypass, videoangiography, ICG, indocyanine green, software analysis, FLOW 800.

\footnotetext{
Correspondencia a:

Dr. José Luis Cuevas

Servicio de Neurocirugía Hospital de Puerto Montt.

Los Aromos 65, Puerto Montt, Chile.

cuevasseguel.joseluis@gmail.com
} 


\section{Introducción}

La videoangiografía con indocianina verde (ICG) es una herramienta que ha tenido gran impacto en neurocirugía vascular desde su introducción en éste ámbito en $2003^{1}$. Su utilidad ha sido descrita principalmente en cirugía de aneurismas, malformaciones arteriovenosas (MAVs), fístulas arteriovenosas (FAVs), cavernomas, bypass y tumores (meningiomas y hemangioblastomas) ${ }^{2}$. La videoangiografía ICG permite una evaluación dinámica del flujo sanguíneo cerebral, no obstante sus resultados son primariamente cualitativos ${ }^{3}$.

EI FLOW 800 (Carl Zeiss Meditec, Oberkochen, Germany) es un software relativamente nuevo e integrado a microscopios, que permite analizar de manera semicuantitativa la información obtenida de la videoangiografía ICG mediante la generación de un mapa visual a color, que identifica la dirección y secuencia del flujo sanguíneo relativo a la vasculatura circundante $^{3-4}$. En el mapa visual, el color rojo representa la afluencia sanguínea inicial, seguido por una escala de colores degradados para las secuencias más retrasadas de flujo sanguíneo. De este modo, se obtiene una evaluación objetiva de la fluorescencia ${ }^{4}$.

El propósito del presente estudio fue describir nuestra experiencia con el uso del análisis de flujo por videoangiografía ICG (FLOW 800) en cirugía vascular cerebral.

\section{Método}

Se presentan dos casos de pacientes tratados quirúrgicamente por el Equipo de Neurocirugía Vascular y Base del Cráneo del Hospital de Puerto Montt entre los años 2016 y 2018, en quienes el uso del análisis de flujo por videoangiografía ICG (FLOW 800) fue determinante para la toma de decisiones quirúrgicas. El Hospital de Puerto Montt cuenta desde el año 2015 con un microscopio (Opmi Pentero 800, Carl Zeiss) con capacidad de realizar videoangiografía ICG y análisis de flujo. Desde el año 2016, este análisis se realiza de rutina en cirugía de MAVs y revascularización cerebral. A la fecha, el FLOW 800 sido utilizado en más de 20 pacientes. A continuación, se muestran dos de nuestros casos más ilustrativos.

\section{Casos clínicos}

\section{Caso 1}

Paciente de 42 años, inicia estudio tras presentar convulsión tónico-clónica generalizada. La resonancia magnética (RM) de encéfalo y angiografía cerebral demostraron la presencia de una MAV frontal derecha de nido compacto, de aproximadamente $2,8 \mathrm{~cm}$, aferentada principalmente por ramos de arteria pericallosa y arteria calloso marginal (también ramos menores de arteria cerebral media), drenaje venoso único a seno sagital y estrecha relación con el área motora (Supplemented Spetzler-Martin grade = 5). Se decidió manejo quirúrgico, para lo cual se planificó un acceso bifrontal con control de aferencias mediales por vía transfalcina contralateral (izquierda) y posterior resección de MAV por derecha. Dado lo compacto del nido, la identificación visual de las aferencias arteriales mediales fue compleja incluso con uso de videoangiografía ICG. El análisis de flujo (FLOW 800), por el contrario, permitió identificar las dos aferencias principales dejando en el medio un vaso con flujo más tardío correspondiente a la vena de drenaje. Una vez identificadas las aferencias arteriales se procedió a su clipaje y se continuó con la resección de la MAV respetando la vena de drenaje hasta el final de la cirugía (Figura 1). El paciente evolucionó favorablemente ( $\mathrm{mRS}=0$ ).

\section{Caso 2}

Pescador de 47 años, quien inicia cuadro de deterioro visual y motor progresivo de 1 año de evolución. Su estudio demostró oclusión de la arteria carótida interna $(\mathrm{ACl})$ derecha asociado a hipoperfusión de hemisferio cerebral derecho (Figura 2). Se decidió realizar bypass cerebral para aumento de flujo (bypass desde arteria temporal superficial a arteria cerebral media; ATS-ACM M2). La intervención consistió en la obtención del injerto de la arteria temporal superficial (ATS), para luego completar una craniotomía pterional. Posteriormente se disecó el valle silviano y se expuso la arteria cerebral media (ACM) en sus segmentos M1 y M2. En ese momento se realizó una videoangiografía ICG que mostró un llene algo enlentecido de las ramas M2 frontales. El análisis de flujo (FLOW 800) confirmó flujo más tardío a nivel frontal M2, por lo que se realizó bypass ATS a rama M2 frontal con sutura $10 / 0$ punto separado. Una segunda videoangiografia ICG y análisis de flujo confirmaron velocidad de llene simétrico en territorio M2 frontal y M2 temporal (Figura 3). La angioTC confirmó la mejoría del llene arterial en el área receptora del bypass y el paciente evolucionó favorablemente $(\mathrm{mRS}=0)$

\section{Discusión}

EI FLOW 800 es una herramienta disponible desde hace pocos años en nuestro medio y probablemente aún subutilizada. A diferencia de la videoangiografía ICG "convencional" nos entrega información objetiva de la secuencia del flujo sanguíneo, mejorando nuestro entendimiento de la fisiopatología arterial, venosa y parenquimatosa durante el acto quirúrgico ${ }^{3}$.

El análisis de flujo es especialmente útil en cirugía de MAVs, donde permite una rápida evaluación de la angioarquitectura del nido malformativo así como de la vena de drenaje. Así mismo, puede mostrar los cambios dinámicos en la vena de drenaje a medida que las aferencias arteriales son ocluidas y puede disminuir potenciales complicaciones por sacrificio venoso precoz. Su limitante principal es que las aferencias profundas no siempre pueden observarse $e^{5-6}$.

En cirugía de revascularización el uso del análisis de flujo también podría tener un rol $^{3}$. Prinz $\mathrm{V}^{7}$, Uchino $\mathrm{H}^{8}$ y $\mathrm{Ye} \mathrm{X}$ et al., han descrito que el FLOW 800 detectó cambios hemodinámicos post bypass de ATS- ACM en términos de mejoría del flujo sanguíneo cerebral ${ }^{7}$. Nuestra experiencia en este tipo de intervención corrobora lo descrito por estos autores, por lo que creemos que el FLOW 800 pudiese tener un rol predictivo 


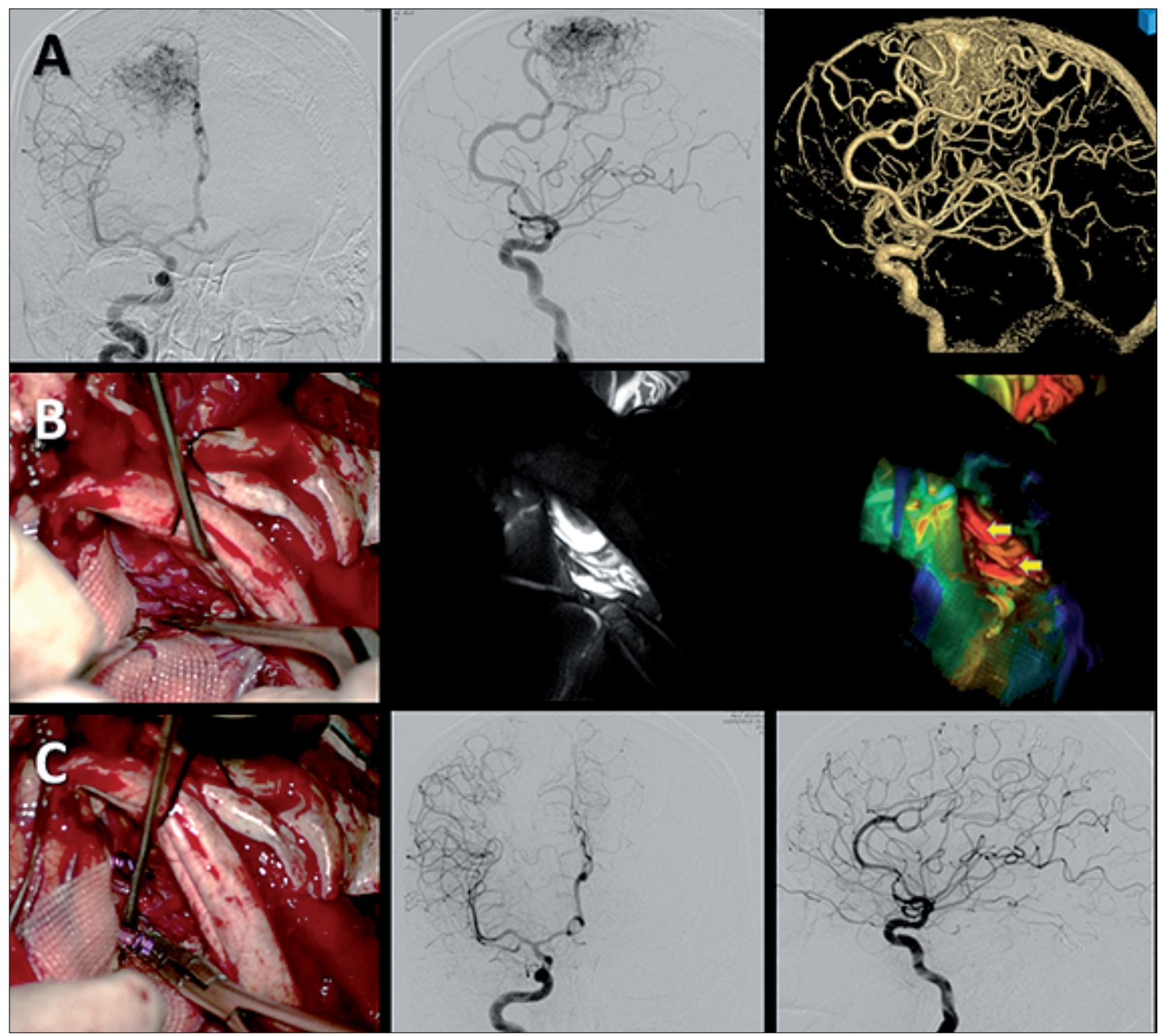

Figura 1. A. Angiografía convencional muestra MAV aferentada principalmente por ramas de arteria cerebral anterior; B. Visión intraoperatoria mediante acceso transfalcino contralateral (izquierda). Tanto la visión quirúrgica como la videoangiografía ICG muestran ovillo malformativo en el que es difícil distinguir vasos aferentes y eferentes. A derecha, el estudio de flujo (Flow 800) permite identificar 2 vasos con llene precoz que corresponden a las aferencias arteriales de la MAV (flechas amarillas) y un vaso único con llene más tardío (vena de drenaje); C. A izquierda se muestran las arterias aferentes clipadas. Al centro y derecha, la angiografía convencional que confirma la exéresis de la MAV.

al confirmar la mejoría en la perfusión regional.

Una de las mayores ventajas que hemos encontrado a esta herramienta es que permite seleccionar de mejor manera la arteria receptora para el bypass. Tal como ha sido descrito por Ashley $\mathrm{W}$ et al. ${ }^{10}$, la selección inadecuada de la arteria recipiente puede resultar en la oclusión del bypass (Error tipo 2). En nuestra práctica, realizamos FLOW 800 de manera sistemática una vez expuesto el valle silviano y la ACM en sus segmentos M1 y M2. Luego analizamos el mapa de colores y utilizamos como arteria receptora la rama M2 con llene más tardío o cuyo territorio regional es el que presenta menor flujo sanguíneo. Ésta técnica ha resultado especialmente efectiva en pacientes sometidos a bypass por isquemia crónica (aumento de flujo), con los que hemos logrado las tasas más altas de permeabilidad.

El análisis de flujo ha sido también descrito para aneurismas complejos ${ }^{1,3}$. En este contexto, puede ser de utilidad para la evaluación de ramas distales con riesgo de estenosis y/o hipoflujo posterior a un clipaje - de manera complementaria a técnicas no invasivas como el flujometro intraoperatorio - indicando la necesidad de modificación del clipaje o realización de bypass ante isquemia persistente.

\section{Conclusiones}

El análisis de flujo (Flow 800) es una herramienta muy útil en neurocirugía vascular. En la experiencia de los autores, puede tener un rol crítico especialmente en MAVs de nidos complejos, donde permite la identificación de vasos aferentes y eferentes, así como cambios dinámicos en las venas de drenaje a medida que las aferencias arteriales son ocluidas. Así mismo, permite una mejor selección de arterias receptoras en cirugía de bypass e incluso pudiese tener un rol pronóstico al demostrar cambios en la perfusión parenquimatosa post revascularización. 


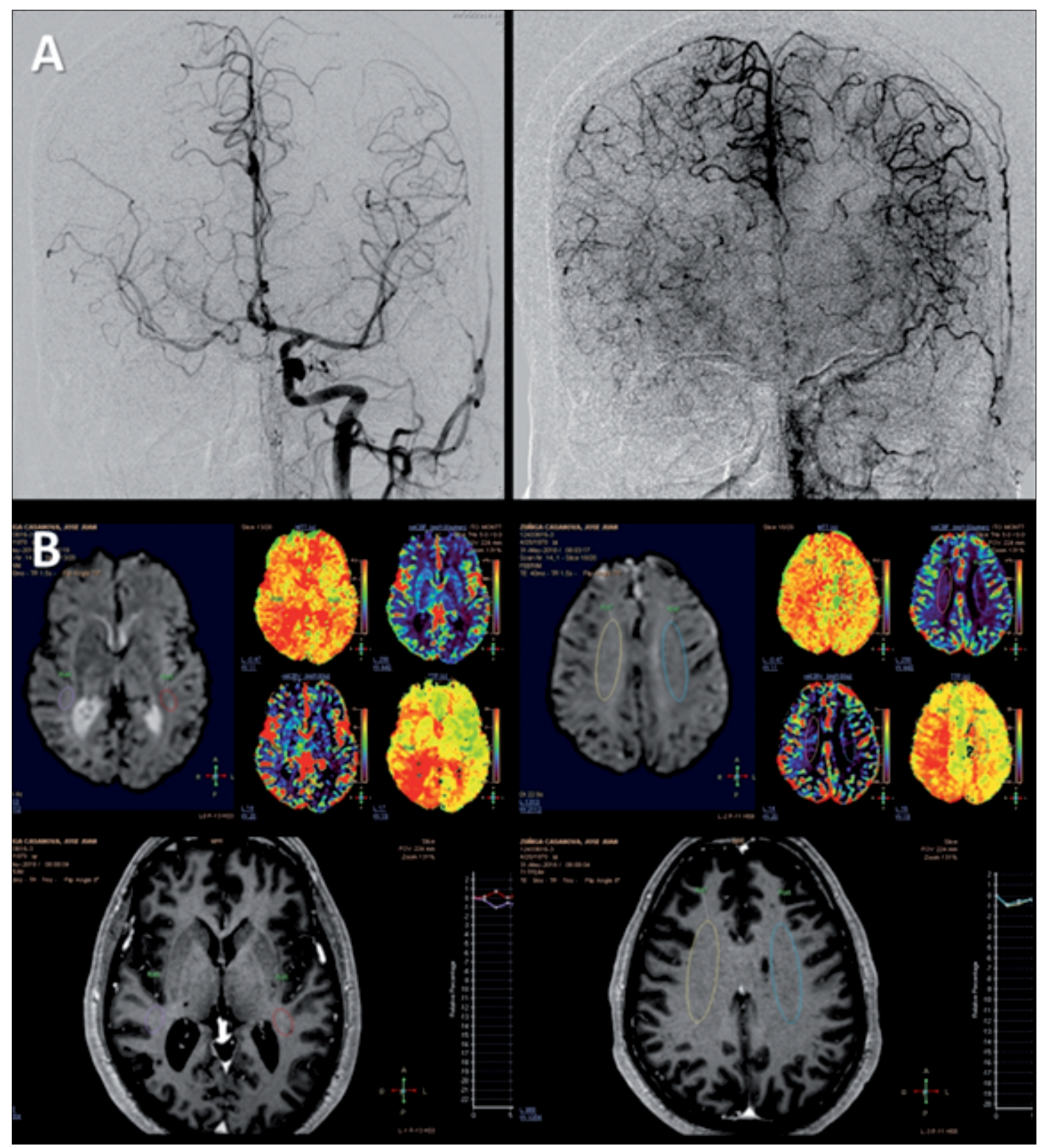

Figura 2. A. Angiografía convencional muestra pobre llene arterial de hemisferio derecho tanto en fase inicial (izquierda) como tardía (derecha); B. Resonancia magnética muestra enlentecimiento de la perfusión del hemisferio derecho (en rojo: aumento del tiempo de transito medio; MTT). 


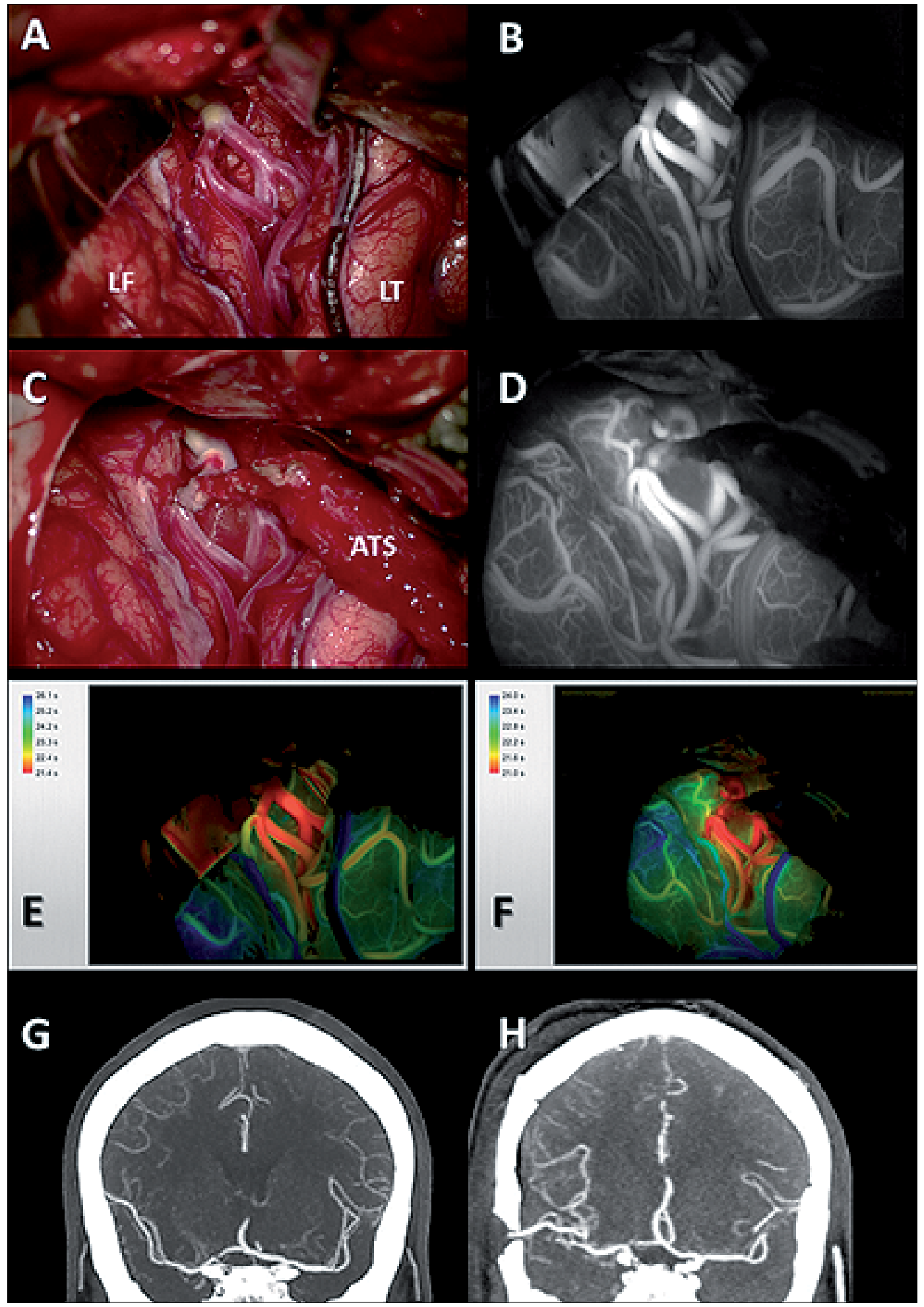

Figura 3. A. Visión intraoperatoria por acceso pterional derecho. Se aprecia el valle silviano expuesto y la ACM en sus segmentos M1 y M2; B. Videoangiografía con ICG muestra flujo sanguíneo basal, con leve retraso de llene en ramas frontales M2. Destaca la menor representación de ramas corticales a nivel frontal; C. Visión intraoperatoria posterior a la confección del bypass; D. Videoangiografía ICG post bypass muestra mayor representación de ramas corticales; E y F. Mapa de colores del estudio de flujo (FLOW 800) pre y post bypass. A izquierda se aprecia llene retrasado de ramas frontales $\mathrm{m}^{2}$ (aproximadamente $1 \mathrm{~s}$ ) con respecto a ramos temporales $\mathrm{m}^{2}$. Posterior al bypass, los tiempos de llene arterial son simétricos, al igual que la perfusión del parénquima; G y H. AngioTC pre y post operatoria, confirman mejoría significativa del llene arterial en hemisferio derecho; LF: Lóbulo frontal; LT: Lóbulo temporal; ATS: Arteria temporal superficial. 


\section{Conflicto de interés:}

El autor no declara conflicto de interés.

\section{Referencias}

1. Raabe A, Beck J, Gerlach R, Zimmermann M, Seifert V. Nearinfrared indocyanine green video angiography: a new method for intraoperative assessment of vascular flow. Neurosurgery. 2003;52:132-139 [discussion: 139].

2. Scerrati A, Della Pepa GM, Conforti G, Sabatino G, Puca A, Albanese A, Maira G, Marchese E, Esposito G. Indocyanine green video-angiography in neurosurgery: A glance beyond vascular applications. Clinical Neurology and Neurosurgery. 2014; 124:106-113.

3. Shah KJ, Cohen-Gadol AA. The Application of FLOW 800 ICG Videoangiography Color Maps for Neurovascular Surgery and Intraoperative Decision Making. World Neurosurg. 2018; E1E12.

4. Jhawar SS, Kato Y, Oda J, Oguri D, Sano H, Hirose Y. FLOW 800-assisted surgery for arteriovenous malformation. J Clin Neurosci. 2011;18: 1556-1557.

5. Fukuda K, Kataoka H, Nakajima N, Masuoka J, Satow T, lihara K. Efficacy of FLOW 800 with indocyanine green videoangiography for the quantitative assessment of flow dynamics in cerebral arteriovenous malformation surgery. World Neurosurg. 2015;83:203-210.

6. Acerbi F, Vetrano IG, Sattin T, Falco J, de Laurentis C, Zattra CM, Bosio L, Rossini Z, Broggi M, Schiariti M, Ferroli P. Use of ICG videoangiography and FLOW 800 analysis to identify the patient-specific venous circulation and predict the effect of venous sacrifice: a retrospective study of 172 patients. Neurosurg Focus 2018; 45 (1):E7.

7. Prinz V, Hecht N, Kato N, Vajkoczy P. FLOW 800 allows visualization of hemodynamic changes after extracranial-to-intracranial bypass surgery but not assessment of quantitative perfusion or flow. Neurosurgery. 2014;10(suppl 2):231-238 [discussion: 238239].

8. Uchino H, Nakamura T, Houkin K, Murata J, Saito H, Kuroda $S$. Semiquantitative analysis of indocyanine green videoangiography for cortical perfusion assessment in superficial temporal artery to middle cerebral artery anastomosis. Acta Neurochir (Wien). 2013;155:599-605.

9. Ye X, Liu XJ, Ma L, Liu LT, Wang WL, Wang S, et al. Clinical values of intraoperative indocyanine green fluorescence video angiography with FLOW 800 software in cerebrovascular surgery. Chin Med J (Engl). 2013;126:4232-4237.

10. Ashley WW, Amin-Hanjani S, Alaraj A, Shin JH, Charbel FT. Flow-assisted surgical cerebral revascularization. Neurosurg Focus. 2008; 24(2): E20. 